Copies of the report: The health of the people of NSW: Report of the Chief Health Officer, 2000 are available from the NSW Department of Health Web site at: www.health.nsw.gov.au/public-health/chorep/ chorep.html. Further details of the evaluation are available on request from Peter Waples, Olympic Planning Unit, NSW Department of Health, Locked Mail Bag 961, North Sydney NSW 2059; or by email at pwapl@doh.health.nsw.gov.au.

\title{
IMPROVING THE QUALITY AND MANAGEMENT OF ABORIGINAL AND TORRES STRAIT ISLANDER HEALTH INFORMATION IN NSW
}

\begin{abstract}
Angela Todd
Manager, Aboriginal Health Information Strategy Unit NSW Department of Health

In 1997 the NSW Department of Health, in collaboration with the Aboriginal Health \& Medical Research Council (AHMRC), developed the Aboriginal Health Information Strategy (AHIS). The Aboriginal Health \& Medical Research Council is the peak body representing member Aboriginal Community Controlled Health Services (ACCHSs) in NSW. This article describes the AHIS, the six key projects it contains, and gives details of the first of these projects: the development of the NSW Aboriginal Health Information Guidelines.
\end{abstract}

\section{ABORIGINAL HEALTH INFORMATION STRATEGY: AIMS AND COORDINATION}

The AHIS aims to improve the quality of information available to support service providers in the planning and delivery of health services for Aboriginal and Torres Strait Islander peoples in NSW. It contributes to the Department's Corporate Plan to:

- promote the delivery of appropriate health services to Aboriginal communities;

- strengthen skills and commitment in quality improvement by increasing capacity to identify and monitor outcomes of care and service delivery, and manage and analyse information;

- enhance information management and analytical skills, including development of new technologies to enhance health services' management.

It also supports the recently released Aboriginal Health Strategic Plan to:

- implement the recommendations of the Multilateral Agreement in relation to the protocols for the collection, use and dissemination of data and information on Aboriginal health.

- improve recording of Aboriginality in all Area Health Service data collections.

- establish co-ordinated disease registers and patient recall systems to enable early intervention, effective management and follow up by Area Health Services and ACCHSs.

Implementation of the AHIS is overseen by the NSW Aboriginal Health Information Management Group (AHIMG), an intersectoral steering committee comprising senior representatives from the following organisations:

- NSW Department of Health

- NSW Area Health Services

- Aboriginal Health \& Medical Research Council

- Aboriginal Community Controlled Health Services

- Aboriginal and Torres Strait Islander Commission

- Commonwealth Office for Aboriginal and Torres Strait Islander Health

- Australian Bureau of Statistics

- Registry of Births, Deaths and Marriages

- Australian Centre for Effective Healthcare.

\section{ABORIGINAL HEALTH INFORMATION STRATEGY PROJECTS}

The AHIS comprises six identified projects:

1. develop an Aboriginal health information agreement, in partnership with the AHMRC, to cover ownership and sharing of data, and confidentiality and privacy guidelines;

2. consult and negotiate with the AHMRC and ACCHSs about their information needs, with a view to possibly assisting in the development and support of 
information systems, and the establishment of common definitions and terms;

3. consult and negotiate with other States and Territories to identify ways that might help improve the quality of information in NSW;

4. collaborate with the Registry of Births, Deaths and Marriages to improve the level of registration of births and deaths, and the Australian Bureau of Statistics to improve population estimates, of Aboriginal peoples in NSW;

5. develop and test programs to improve the underrecording of Aboriginality in mainstream health information systems;

6. identify and assess ways of obtaining supplementary information on the health of Aboriginal peoples in NSW.

\section{Project 1: NSW Aboriginal Health Information Guidelines}

The first priority of the AHIS was Project 1: develop an Aboriginal health information agreement in partnership with the AHMRC to cover ownership and sharing of data, and confidentiality and privacy guidelines. Work on the development of an agreement commenced in January 1997 and, following lengthy consultation, culminated in the development of the NSW Aboriginal Health Information Guidelines, ${ }^{1}$ which were launched by the Minister for Health in August 1998.

The guidelines are the first of their kind in Australia. They aim to ensure consistency and good practice in the management of health and health-related information about Aboriginal and Torres Strait Islander peoples in NSW. In particular, the guidelines provide a framework and set of principles for the collection, ownership and use of information, as well as taking action on issues of confidentiality, privacy and consent. The guidelines briefly consider research involving Aboriginal peoples and communities, however there are national guidelines that provide more detailed information to assist researchers in this regard. ${ }^{2}$

Since the guidelines were released, staff feedback has highlighted the need to clarify how the guidelines can be applied to various routine information processes. As a result, and to facilitate ongoing implementation of the guidelines, a simple flow-chart has been developed (see Figure 6). This diagram relates to all information collections owned or held by NSW Health. A copy of the guidelines and this flow-chart can be downloaded from the AHIS Intranet site. A copy of the guidelines alone, minus the flow-chart, can be downloaded from the AHIS Web site (see box below).

A review of the guidelines is scheduled for September 2000. The Commonwealth Department of Health and Aged Care has recently commenced work on the development of national data protocols for Aboriginal and Torres Strait Islander health information. The review of the guidelines will provide an opportunity to incorporate any necessary changes arising from the national project, but also any changes identified by stakeholders as a result of practical application of, and experience with, the guidelines. This will be an important opportunity for NSW Health staff to provide feedback.

\section{CONCLUSION}

The guidelines are a useful resource for staff working in the provision of health services for Aboriginal and Torres Strait Islander peoples, and for information custodians, information managers, epidemiologists, researchers and Research Ethics Committees.

Since the release of the guidelines, work has continued on the remaining AHIS projects. Priority is currently being given to Project 5: improving the under-recording of Aboriginal and Torres Strait Islander origin information in mainstream health information systems. Details of this work will be provided in a future publication of The Bulletin.

\section{REFERENCES}

1. NSW Department of Health. NSW Aboriginal Health Information Guidelines, State Publication No. (AHB) 980128. Sydney: NSW Department of Health, 1998.

2. National Health and Medical Research Council. Guidelines on Ethical Matters in Aboriginal and Torres Strait Islander Health Research. Canberra: AGPS, 1991. it:

The guidelines can be viewed and downloaded from the NSW Department of Health AHIS Web site at www.health.nsw.gov.au/iasd/imcs/ahisu. Free copies of the guidelines can also be obtained from the Better Health Centre by telephone on (02) 9816 0452, quoting State Health Publication number AHB 980128. Further information and inquiries can be directed to Angela Todd, Aboriginal Health Information Strategy, NSW Department of Health at: antodd@doh.health.nsw.gov.au. 
NSW ABORIGINAL HEALTH INFORMATION GUIDELINES (AHIG):

WHENTO APPLY THEM INTHE COLLECTION, ACCESS, USE AND PUBLISHING OF ABORIGINAL ANDTORRES STRAIT ISLANDER INFORMATION

Note: The AHIG and this diagram should be read in conjunction with the NSW Health Information Privacy Code of Practice, and the NSW Information Privacy Principles.

This diagram relates to all NSW Health data collecitons.

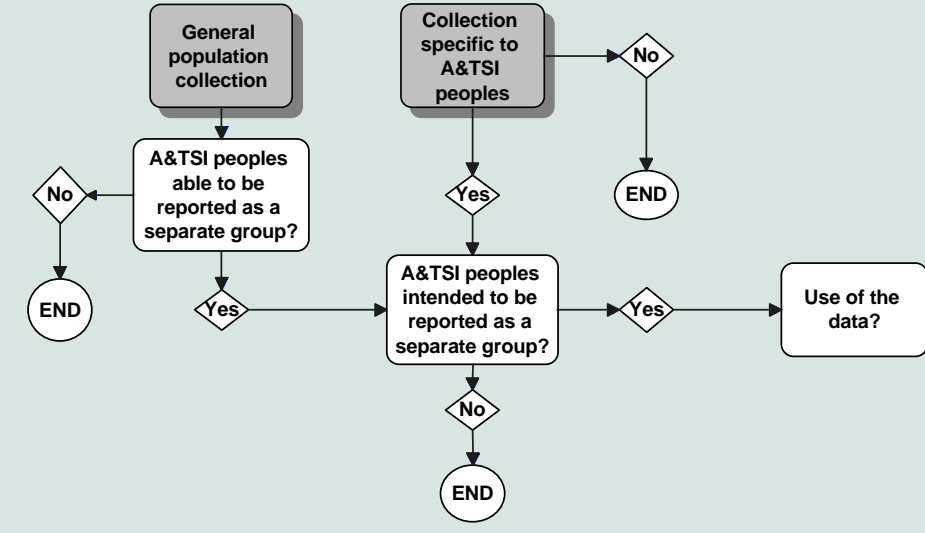

Glossary

Aboriginal Health \& Medical Research Council (AH\&MRC)

peak body representing Aboriginal Community Controlled Health Services in NSW. Commonwealth-State reporting

includes Australian Health Care Agreement, National Health Information Agreement (

Ol request

application by a third party for access to information determined under the FOI Act. Funding \& performance agreements

includes agreements and contracts with other parties for the provision of services, for example, NGOs.

Internal Department-AHS reporting

includes financial, audit, Area Health Service performance agreements, etc.

Internal report to other government agency

includes Treasury, Department of Community Services, the Ombudsman, the

Published report by $D O H, A H S$, other government agency

includes Chief Health Officer's Report, Mothers and Babies Annual report, reports

released on the Internet.

Research study

defined as systematic investigation for the purpose of adding to generalised

knowledge pertaining to human behaviour and includes epidemiological research

(Section 8 Privay Guidelines).

Statutory \& other legal requiremen

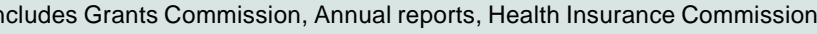

Guardianship Board and Protective Office, NSW Cancer Registry, subpoenas.

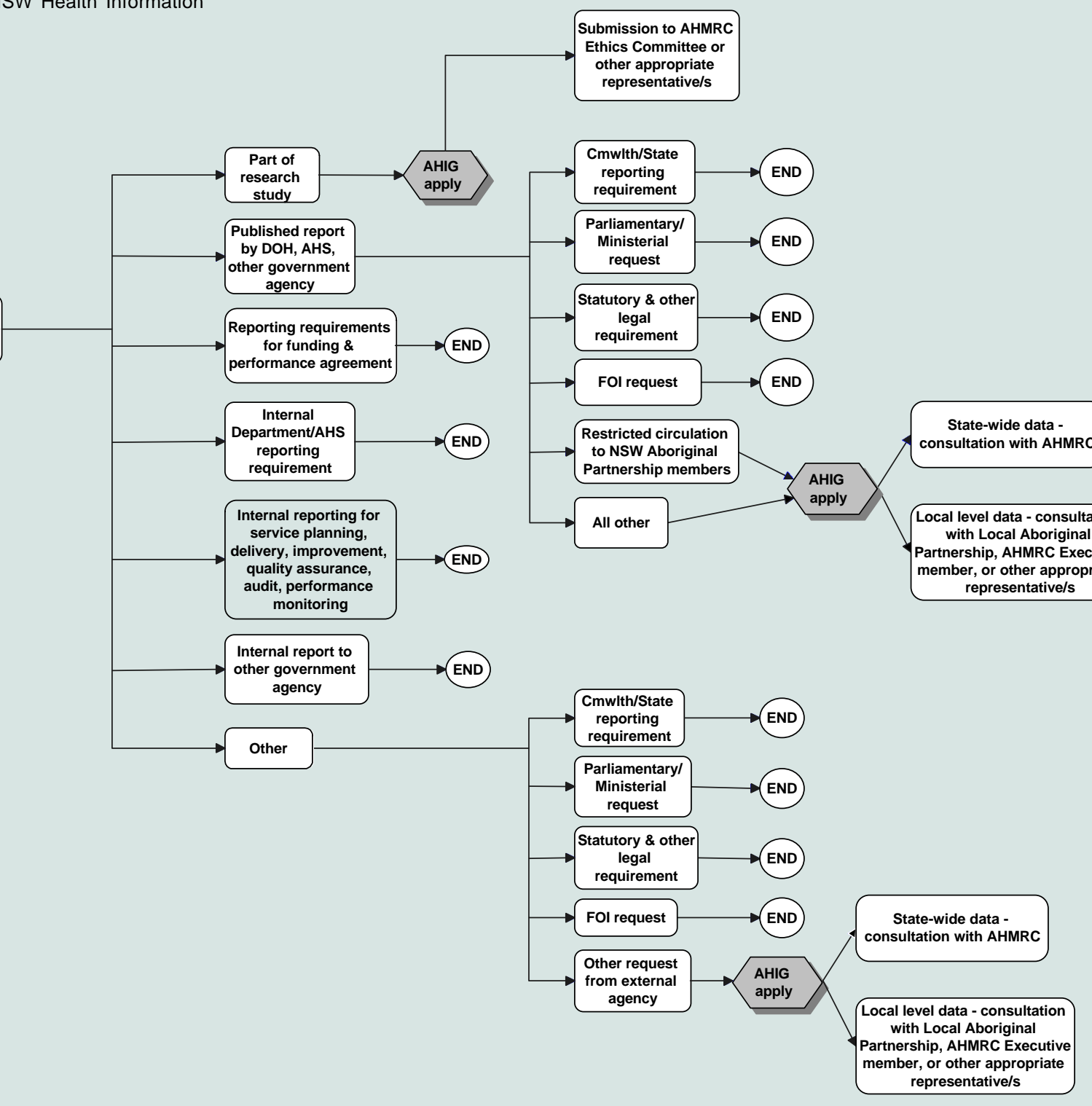

